\title{
Validity and Reliability of the Japanese Version of the Rome III Diagnostic Questionnaire for Irritable Bowel Syndrome and Functional Dyspepsia
}

\author{
Motoyori Kanazawa, ${ }_{1}{ }^{*}$ Shigemi Nakajima, ${ }^{2}$ Tadayuki Oshima, ${ }^{3}$ William E Whitehead, ${ }^{4}$ Ami D Sperber, ${ }^{5}$ Olafur S Palsson, ${ }^{4}$ \\ Douglas A Drossman, ${ }^{4}$ Hiroto Miwa, ${ }^{3}$ and Shin Fukudo ${ }^{1}$ \\ ${ }^{1}$ Department of Behavioral Medicine, Tohoku University Graduate School of Medicine, Sendai, Japan; ${ }^{2}$ Department of Medicine, \\ Gastroenterology and Health Care, Japan Community Health care Organization Shiga Hospital, Otsu, Shiga, Japan; ${ }^{3}$ Division of Upper \\ Gastroenterology, Department of Internal Medicine, Hyogo College of Medicine, Nishinomiya, Hyogo, Japan; ${ }^{4}$ Center for Functional GI and \\ Motility Disorders, University of North Carolina at Chapel Hill, Chapel Hill, NC, USA; and ${ }^{5}$ Department of Gastroenterology, Tel Aviv Medical \\ Center, Levahim, Israel
}

\begin{abstract}
Background/Aims
Reliable diagnostic instruments for measuring the presence of functional gastrointestinal disorders based on the Rome III criteria have been lacking in Japan. The aims of the present study were to translate and validate the Rome III diagnostic questionnaire which was widely used in Western countries.

\section{Methods}

The original version of Rome III diagnostic questionnaire was translated from English into Japanese through 3 independent forward translations, resolution, back translation and reconciliation of the differences. Forty-nine patients with irritable bowel syndrome (IBS), 32 patients with functional dyspepsia (FD) and 56 subjects without any current Gl symptoms as controls were recruited from three hospitals located in different regions of Japan and completed the IBS and FD diagnostic modules twice within 14 days. Kappa statistic was used to assess test-retest reliability. The sensitivity and specificity of each diagnostic module for distinguishing IBS or FD patients from controls was tested.
\end{abstract}

Received: January 23, 2015 Revised: May 1, 2015 Accepted: May 5, 2015

(c) This is an Open Access article distributed under the terms of the Creative Commons Attribution Non-Commercial License (http://creativecommons. org/licenses/by-nc/4.0) which permits unrestricted non-commercial use, distribution, and reproduction in any medium, provided the original work is properly cited.

*Correspondence: Motoyori Kanazawa, MD, PhD

Department of Behavioral Medicine, Tohoku University Graduate School of Medicine, 2-1 Seiryo, Aoba, Sendai 980-8575, Japan Tel: +81-22-717-7655, Fax: +81-22-717-7655, E-mail: m-kanazawa@med.tohoku.ac.jp

Financial support: This study was supported by a Grant-in-Aid for Scientific Research from the Ministry of Education, Science, and Culture of Japan, and a Grant-in-Aid for Scientific Research from the Ministry of Health, Welfare, and Labor of Japan.

Conflicts of interest: The authors declare that they have no conflicts of interest except for Shin Fukudo, Douglas Drossman, William Whitehead, and Olafur Palsson. Professor Fukudo received a research grant from Kao and Astellas. He also received lecture fees from Abbott Japan, Dainippon-Sumitomo, and Astellas. Professor Drossman is a President of the Rome Foundation that created the Rome III criteria and he receives a stipend for this work. He also receives royalties for publication of the Rome III book. Professors Whitehead and Palsson received research grants from Ironwood Pharmaceuticals, Takeda Pharmaceuticals, and Salix Pharmaceuticals. Professor Whitehead is a consultant to Ono Pharma USA

Author contributions: Motoyori Kanazawa participated in study design, data collection, data analysis, and preparation of the manuscript; Shigemi Nakajima participated in data collection; Tadayuki Oshima participated in data collection; William E Whitehead participated in study design and preparation of the manuscript; Ami D Sperber, Olafur S Palsson and Douglas A Drossman participated in preparation of the manuscript; Hiroto Miwa participated in study design and data collection; and Shin Fukudo participated as the guarantor of this paper, and he participated in study design, data collection, data analysis, and manuscript preparation. ORCID: Motoyori Kanazawa, http://orcid.org/0000-0003-1953-3336. 


\section{Results}

Median kappa statistics were 0.63 for the translated IBS diagnostic module and 0.68 for the FD module. The sensitivity, specificity, and positive predict value of the IBS module against physician diagnosis was $61.2 \%, 100 \%$, and $100 \%$ and those of the FD module was $53.2 \%, 98.2 \%$, and $94.4 \%$, respectively. Meanwhile, IBS patients were significantly more likely to report blood in stools compared to controls $(18.4 \%$ vs $1.8 \%, P<0.01)$.

\section{Conclusions}

The IBS and FD diagnostic modules on the Japanese version of the Rome III diagnostic questionnaire are valid and reliable. Further studies are warranted to elucidate the diagnostic utility of the red flag questionnaire.

(J Neurogastroenterol Motil 2015;21:537-544)

\section{Key Words}

Dyspepsia; Functional gastrointestinal disorders; Irritable bowel syndrome; Japan; Questionnaire; Translations

\section{Introduction}

Rome III diagnostic criteria based on subjective gastrointestinal (GI) complaints are the most widely used criteria to diagnose functional gastrointestinal disorders (FGIDs). ${ }^{1}$ Irritable bowel syndrome (IBS) and functional dyspepsia (FD) are the most common FGIDs both in the Western and the Eastern countries. ${ }^{2,3}$ The Rome committee critically considered the available evidence, and multinational expert opinion, when they revised the former Rome II diagnostic criteria ${ }^{4}$ and updated diagnosis and treatment recommendations.

The Rome III diagnostic criteria for FGIDs provide a framework for symptom-based diagnosis. The Rome committee proposed that subtyping of IBS should be based on frequency and consistency of stools, ${ }^{5}$ which can be assessed with the aid of the Bristol Stool Form Scale. ${ }^{6}$ The committee also proposed that the newly defined entities of (1) meal-induced dyspeptic symptoms (postprandial distress syndrome, PDS), and (2) epigastric pain (epigastric pain syndrome, EPS) should be treated as subgroups of FD for pathophysiological and therapeutic research purposes. ${ }^{3}$

The Rome Foundation developed a questionnaire for the Rome III diagnostic criteria for FGIDs. They also validated the questionnaire to assess the test-retest reliability of the questionnaire for all diagnoses combined and for individual questions, and to test the sensitivity and specificity of the questionnaire for identifying medically diagnosed patients with FGIDs. The Rome III diagnostic questionnaire for IBS contains 10 items and the one for FD contains 18 items; answers to questions are on an ordinal scale with individual frequency thresholds for each question. ${ }^{7}$ While "red flag" questions are not part of the diagnostic questionnaire, these features, if present, should prompt the clinician to consider further investigations to exclude other serious medical conditions. Briefly, the "red flag" questions include history for the past 3 months of fever, weight loss, cancer in family members, blood mixed with stool, anemia and change in bowel habit after age $50 .{ }^{8}$ Red flags may be useful for identifying patients who require additional diagnostic evaluation in the clinical settings. However, it has been investigated that incorporating them into the Rome II criteria did not improve sensitivity and resulted in too many missed diagnoses of IBS. ${ }^{8}$

Previously, we have developed and validated the Japanese translated version of the Rome II modular questionnaire based on the former diagnostic criteria for IBS. ${ }^{9}$ There are a few studies to investigate prevalence of FGIDs using the Rome III criteria in Japan. ${ }^{10,11}$ However, reliable diagnostic instruments for measuring the presence of FGIDs based on the Rome III criteria, comparable to those used in Western countries, have been lacking in Japan. To make cross-cultural comparisons possible, it is indispensable to develop common diagnostic measures for FGIDs. ${ }^{12}$ The aims of the present study were to translate the Rome III diagnostic questionnaire into Japanese and to validate them in Japanese patients with IBS and FD. In addition, we investigated whether the utility of red flag symptoms that can be incorporated into the Japanese instruments.

\section{Materials and Methods}

\section{Translation}

The Rome III diagnostic questionnaire was developed as a 
diagnostic instrument for functional GI disorders according to the Rome III diagnostic criteria. ${ }^{7}$ All answers in the Rome III diagnostic questionnaire were in ordinal scale with individual frequency thresholds except for 22 of 81 items which had a "yes" or "no" response. A score was given to each of the responses for ordinal scaled items ( 0 , never; 1 , sometimes; 2 , often; 3 , most of the time; and 4, always). There were 10 items for IBS diagnosis, 18 items for FD diagnosis, and 6 items for the "red flag" symptoms/conditions (blood in stools, unintended weight loss of over 10 pounds $(4.5 \mathrm{~kg}$ ), fever, symptom onset after age 50 , and family histories of any GI cancer and inflammatory bowel disease) were used.

A group of the Japanese co-investigators (S.F. and M.K.) independently translated the English version of the Rome III diagnostic questionnaire into the Japanese language. Meanwhile, an investigator (S.N.) independently translated it in the same way to resolve the potential incongruity of the linguistic expressions between the Eastern and Western areas in Japan. After the descriptions of each item had been discussed with specialists, the Japanese version of the instruments was then counter-translated into English by a native speaker of English. This back-translated version was sent to the Rome Committee, compared with the original versions, and discussed to confirm that the Japanese-translated versions were comparable to the English versions of the Rome III diagnostic questionnaire. Then, the Japanese version of the instruments was approved by the authors of the original English versions.

\section{Subjects}

Forty-nine consecutive patients (21 females, mean age 40.9 years) clinically diagnosed by physicians as having IBS according to Rome III diagnostic criteria, and 32 patients with FD (26 females, mean age 53.8 years) diagnosed according to Rome III were enrolled from GI clinics in Tohoku University Hospital, Hyogo College of Medicine Hospital, and Japan Community Health care Organization Shiga Hospital, Japan. Clinical examinations including upper and/or lower GI endoscopy had been carefully performed and no abnormal findings to explain GI symptoms had been detected when diagnosed as FD or IBS. Fifty-six subjects ( 32 females, mean age 51.4 years) who visited at the same hospitals for an annual health check $(n=31)$ or treatment of mild hypertension/hyperlipidemia $(n=25)$ but did not have any current GI complaints and any past history of abdominal surgeries except for appendectomy were also recruited as controls. Control subjects were carefully diagnosed as not having any FGID by their physicians.

All the participants were Japanese and were 18 years of age or older. They did not have any organic GI diseases or any other severe physical or psychiatric complications. Informed consent was obtained from all the subjects. However, a study ID was assigned to insure that the investigators could not identify any subjects. This study was approved by the Ethics Committees of Tohoku University Graduate School of Medicine, Hyogo College of Medicine and Japan Community Health care Organization Shiga Hospital.

\section{Severity of Irritable Bowel Syndrome Symptoms}

The IBS symptom severity scale (IBS-SSS) was developed and is widely used in Western countries to assess the severity of lower GI symptoms and the degree to which the quality of life is impaired by IBS. ${ }^{12}$ This instrument has five items, and the total score can range from 0 to 500 . The IBS-SSS scores severity and frequency of abdominal pain, severity of abdominal distension, dissatisfaction of bowel movements, and the interference with life, with a 100 -point scale $(0$, none and 100 , worst) for each question. With the possible exception of the bowel dissatisfaction item, these symptom questions are appropriate for characterizing the severity of symptoms in FD as well as the severity of IBS. In the original English version, IBS is graded as mild (75-174), moderate (175-299), or severe (300-500) on the basis of clinical observations of IBS patients. ${ }^{13}$ Our group previously developed the Japanese version of IBS-SSS and has confirmed the reliability and validity of this questionnaire.

\section{Procedure}

Physician diagnosis for IBS, FD, and control was coded into numbers. The diagnostic code and a study ID were written on a cover page of the first questionnaire for each subject in advance. The first questionnaire containing all 81 questions on the original complete version plus the IBS-SSS was completed by each participant during a clinic visit. Fourteen days later, all subjects were asked to complete a second questionnaire and return it by post to test for reproducibility of answers. The follow-up questionnaire included only the modules for diagnoses of IBS (10 items, the IBS diagnostic module) and FD (18 items, the FD diagnostic module).

\section{Statistical Methods}

The test-retest reliability of the questionnaire was judged 
with the use of the Kappa statistic to assess concordance between questionnaire responses on two separate occasions. The Pearson's chi-square tests were used to assess the agreement between a positive diagnosis by the Japanese version of Rome III diagnostic questionnaire and a clinical diagnosis assessed by their physicians. The sensitivity, specificity, and positive predictive value of the diagnostic questionnaire for discriminating IBS patients from controls, and for discriminating $\mathrm{FD}$ patients from controls were also analyzed. One-way analysis of variance (one-way ANOVA) was used to assess difference in the symptom severity scores between the clinical diagnostic groups. The discriminant validity was measured by comparing the grade of severity based on the IBS-SSS between subjects with Rome-positive and Rome-negative IBS using the nonparametric correlation coefficient, Kendall's tau-b. The proportion of each red flag symptom/condition was compared using the Fisher's exact test between the clinical diagnostic groups.

\section{Results}

No apparent modifications were required to resolve inconsistencies between the forward translations into the Japanese language. The translation prepared by S.F. and M.K. was primarily selected as the final version at this stage. When comparing the back translation to the original English version minor adjustments in the choice of words for "bothersome" (questions 26 and 39), "retching" (question 36), and "several" (question 79) were made in the Japanese translation. The Rome foundation board approved the completed translation in January 2010 (Supplementary Figure).

Characteristics of the sample are shown in Table 1. There was no difference in gender ratio among the groups. Patients with IBS were significantly younger in age compared with FD patients or control subjects. Each patient with IBS was subtyped as IBS with diarrhea $(\mathrm{n}=28)$, IBS with constipation $(\mathrm{n}=9)$, mixed IBS $(n=11)$, or unclassified IBS $(n=1)$ by their physician according to dominant stool consistencies. Each patient with FD was subtyped as postprandial distress syndrome (PDS, $\mathrm{n}=$ 25 ) or epigastric pain syndrome (EPS, $n=7$ ) by their physician based on the upper GI symptoms. None of FD patients was diagnosed as overlap of PDS and EPS.

The total score on the IBS-SSS in patients with IBS was significantly higher than FD patients or control subjects $(P<0.01$, respectively; Table 1). IBS patients also showed significantly

Table 1. Characteristics of Samples

\begin{tabular}{|c|c|c|c|}
\hline & Controls & FD & IBS \\
\hline Number (females) & $56(32)$ & $32(26)$ & $49(21)$ \\
\hline Age $(y r)$ & $51.4 \pm 2.2$ & $53.8 \pm 3.0$ & $40.9 \pm 2.3^{\mathrm{a}, \mathrm{c}}$ \\
\hline \multicolumn{4}{|l|}{ Number of subtypes of IBS } \\
\hline Diarrhea & - & - & 28 \\
\hline Constipation & - & - & 9 \\
\hline Mixed & - & - & 11 \\
\hline Unclassified & - & - & 1 \\
\hline \multicolumn{4}{|l|}{ Number of subtypes of FD } \\
\hline PDS & - & 25 & - \\
\hline EPS & - & 7 & - \\
\hline \multicolumn{4}{|l|}{ Number of types of non-GI controls } \\
\hline Annual health check & 31 & - & - \\
\hline Mild hypertension/hyperlipidemia & 25 & - & - \\
\hline IBS-SSS total score & $30 \pm 7$ & $192 \pm 16^{\mathrm{a}}$ & $252 \pm 16^{\mathrm{a}, \mathrm{c}}$ \\
\hline Abdominal pain severity & $1 \pm 1$ & $21 \pm 4^{\mathrm{a}}$ & $45 \pm 4^{\mathrm{a}, \mathrm{c}}$ \\
\hline Abdominal pain frequency & $0.1 \pm 0.0$ & $2.7 \pm 0.6^{\mathrm{a}}$ & $4.3 \pm 0.5^{\mathrm{a}, \mathrm{b}}$ \\
\hline Abdominal bloating & $2 \pm 1$ & $42 \pm 6^{\mathrm{a}}$ & $38 \pm 5^{\mathrm{a}}$ \\
\hline Bowel dissatisfaction & $18 \pm 4$ & $55 \pm 6^{\mathrm{a}}$ & $65 \pm 4^{\mathrm{a}}$ \\
\hline Interference with life & $8 \pm 3$ & $46 \pm 6^{a}$ & $62 \pm 4^{a}$ \\
\hline
\end{tabular}

Characteristics of each group were shown.

FD, functional dyspepsia; IBS, irritable bowel syndrome; PDS, postprandial distress syndrome; EPS, epigastric pain syndrome; GI, gastrointestinal; IBS-SSS, IBS symptom severity scale.

Data were expressed as mean \pm SEM, ${ }^{\mathrm{a}} P<0.01$ vs Controls; ${ }^{\mathrm{b}} P<0.05,{ }^{\mathrm{c}} P<0.01$ vs FD. 
higher scores than FD patients on IBS-SSS items measuring the intensity and frequency of abdominal pain (Table 1). However, there was no difference in the scores for abdominal bloating, bowel dissatisfaction or interference with life on the IBS-SSS between IBS and FD groups. Control subjects had significantly lower symptom scores for all subscales and the overall symptom severity score on the IBS-SSS compared with IBS or FD patients $(P<$ 0.01 , respectively; Table 1 ).

With respect to the test-retest reliability for the Rome III diagnostic questionnaire, median kappa statistic for the IBS diagnostic module was 0.63. Median kappa statistic for the FD module was 0.68 .

The instrument for the symptom-based IBS diagnostic questionnaire was in modest agreement with physician diagnosis; sensitivity of $61.2 \%$ and specificity of $100 \%$ for distinguishing IBS from health (Table 2). The positive predictive value (PPV) on the IBS module was $100 \%$. When applied the functional bowel disorders (FBD) module on the Rome III criteria ${ }^{1}$ instead of the IBS module, the sensitivity and specificity were $91.8 \%$ and $50.0 \%$. PPV on the FBD module was $61.6 \%$.

The FD module was also in modest agreement with physician diagnosis; sensitivity of $53.2 \%$, specificity of $98.2 \%$ and PPV of $94.4 \%$ for distinguishing FD from health (Table 3). None of subjects with hypertension or hyperlipidemia ( $\mathrm{n}=25)$ was identified as IBS or FD. It is interesting to note that 19 of 49 (39\%)

Table 2. Agreement Between Physician Diagnosis and the Irritable Bowel Syndrome Diagnostic Module

\begin{tabular}{lccc}
\hline \multicolumn{1}{c}{ Diagnosis } & IBS & Controls & Total \\
\hline Rome IBS $(+)$ & 30 & 0 & 30 \\
Rome IBS $(-)$ & 19 & 56 & 75 \\
Total & 49 & 56 & 105 \\
\hline
\end{tabular}

Sensitivity of the irritable bowel syndrome (IBS) diagnostic module was $61.2 \%$, specificity (100\%), and positive predict value (100\%).

Data were expressed as number.

Table 3. Agreement Between Physician Diagnosis and the Functional Dyspepsia Diagnostic Module

\begin{tabular}{lccc}
\hline \multicolumn{1}{c}{ Diagnosis } & FD & Controls & Total \\
\hline Rome FD $(+)$ & 17 & 1 & 18 \\
Rome FD $(-)$ & 15 & 55 & 70 \\
Total & 32 & 56 & 88 \\
\hline
\end{tabular}

Sensitivity of the functional dyspepsia (FD) diagnostic module was $53.2 \%$, specificity (98.2\%), and positive predict value (94.4\%).

Data were expressed as number. patients with IBS were also diagnosed as FD when using the Rome III diagnostic questionnaire. On the other hand, 3 of 32 (9\%) patients with FD were overlapping IBS.

When the score on the IBS-SSS for all the participants was graded as mild graded as mild (75-174), moderate (175-299), or severe (300-500), ${ }^{13}$ subjects with Rome-positive IBS $(\mathrm{n}=33)$ significantly had more severe symptoms compared to those who were not diagnosed as IBS (Rome-negative IBS, $\mathrm{n}=100$ ) using the diagnostic questionnaire (Kendall's tau-b $=0.45, P<$ 0.001 ; Table 4).

With respect to frequencies of the red flags, IBS patients were significantly more likely to report blood in stools (18.4\% vs $1.8 \%, P<0.01$; Fisher's exact test) (Table 5), unintended weight loss $(10.2 \%$ vs $0 \%, P<0.05)$ and new bowel symptom onset after age $50(40.0 \%$ vs $10.8 \%, P<0.05)$ compared to controls. FD patients were more likely to report only unintended weight loss $(12.5 \%$ vs $0 \%, P<0.05)$ compared to controls. There was no significant difference in frequency of fever or fam-

Table 4. Discriminative Validity With the Irritable Bowel SyndromeSymptom Severity Scale in the Rome III Diagnostic Questionnaire (Adapted from Francis et $\mathrm{al}^{13}$ )

\begin{tabular}{lccccc}
\hline IBS-SSS Score & $0-74$ & $75-174$ & $175-299$ & $300-500$ & Total \\
\hline Rome IBS $(+)$ & 0 & 7 & 15 & 11 & 33 \\
Rome IBS $(-)$ & 49 & 22 & 20 & 9 & 100 \\
Total & 49 & 29 & 35 & 20 & 133 \\
\hline
\end{tabular}

Score on the irritable bowel syndrome-symptom severity scale (IBS-SSS) was graded as mild (75-174), moderate (175-299), or severe (300-500). Subjects with Rome-positive IBS had more severe symptoms compared to those who were not diagnosed as IBS $(P<0.001)$.

Data were expressed as number.

Table 5. Frequencies of the "Red Flag" Symptoms

\begin{tabular}{lccc}
\hline \multicolumn{1}{c}{ Frequency $(\%)$} & $\begin{array}{c}\text { Controls } \\
(\mathrm{n}=56)\end{array}$ & $\begin{array}{c}\text { FD } \\
(\mathrm{n}=32)\end{array}$ & $\begin{array}{c}\text { IBS } \\
(\mathrm{n}=49)\end{array}$ \\
\hline Blood in stools & 1.8 & 12.5 & $18.4^{\mathrm{b}}$ \\
Unintended weight loss & 0 & $12.5^{\mathrm{a}}$ & $10.2^{\mathrm{a}}$ \\
Fever & 0 & 0 & 6.0 \\
Bowel symptom onset after age 50 & 10.8 & 30.0 & $40.0^{\mathrm{a}}$ \\
Family history of any GI cancer & 28.6 & 28.1 & 25.5 \\
Family history of IBD & 0 & 3.4 & 4.2 \\
\hline
\end{tabular}

Frequencies of the self-reported symptoms or family histories which would suggest further evaluation to exclude organic gastrointestinal (GI) diseases were shown.

$\mathrm{FD}$, functional dyspepsia; IBS, irritable bowel syndrome; IBD, inflammatory bowel disease.

${ }^{\mathrm{a}} P<0.05,{ }^{\mathrm{b}} P<0.01$ vs Controls (Fisher's exact test). 
ily history of any GI cancer or inflammatory bowel disease between the groups.

\section{Discussion}

The Rome III diagnostic questionnaire was translated into Japanese using the guidelines recommended by the Rome Foundation, and the translated instrument was reviewed and approved by the Rome Foundation. In this study we evaluated the validity of the Japanese-translated Rome III diagnostic questionnaire by examining the concordance between questionnaire based diagnoses and clinical diagnoses in patients with IBS and FD attending gastroenterology out-patient clinics in Japan. We found modest sensitivity for both the IBS questionnaire module (61.2\%) and the FD module (53.2\%). However, the specificity (100\% for IBS, $98.2 \%$ for FD) and the PPV (100\% for IBS and $94.4 \%$ for FD) were excellent. This suggests that clinician-diagnosed FD may be missed by the Japanese Rome III diagnostic questionnaire, but few subjects without FD will be incorrectly classified as IBS or FD cases.

We compared the sensitivity and specificity of the Japanese Rome III Diagnostic Questionnaire to the sensitivity and specificity of the Rome III Diagnostic Questionnaire in other languages. The original English language version of the questionnaire was tested in 328 patients with IBS diagnosed by gastroenterologists and 554 healthy controls, and sensitivity-defined as the proportion of medically diagnosed IBS patients identified by the questionnaire-was $71 \%$, while specificity-defined as the proportion of healthy controls correctly classified as non-IBS-was $88 \% .{ }^{14}$ A translation of the Rome III questionnaire into the Malaysian language similarly showed a sensitivity of $81 \%$ and specificity of $100 \%$ for discriminating 31 clinically diagnosed IBS patients from 31 healthy controls. ${ }^{15}$ A translation of the Rome III questionnaire into Portuguese discriminated medically diagnosed patients with FD from health controls with a sensitivity of $91 \%$ and specificity of $95 \% .{ }^{16}$ It has been reported that a sensitivity of the modified Rome III Diagnostic Questionnaire differs among the Asian countries, ${ }^{17}$ suggesting that socio-cultural perspective and/or the linguistic nuances might be taken into account to translate the original questionnaire into different languages.

In studies which have tested the ability of the Rome III questionnaire to discriminate medically diagnosed IBS patients from patients with other gastrointestinal diagnoses such as inflammatory bowel disease or FD rather than discriminating them from healthy controls, performance has been more modest: In one study using the Rome II criteria, the questionnaire's sensitivity-defined as the ability to discriminate IBS patients from those with other gastrointestinal diagnoses-was $60 \%$ and specificity was $56 \% .^{18}$ In a Norwegian study, which tested the discrimination of IBS from patients with other causes of abdominal pain, sensitivity was $39 \%$ and specificity was $63 \%$. ${ }^{19}$ On the other hand, using with the Korean version of the Rome III questionnaire, the sensitivity was $77.8 \%$ for the IBS module and $70.0 \%$ for the FD module and specificity was $81.6 \%$ and $63.0 \%$ when discriminated clinical IBS or FD patients from subjects who visited at gastroenterologists for any other reasons. ${ }^{20}$ These data show that the sensitivity and specificity of the Japanese Rome III Diagnostic Questionnaire is as good as the English original and other translations of the Rome III questionnaire when similar methodologies are used to evaluate sensitivity and specificity. In this study, test-retest reliability over an interval of two weeks was acceptable with median kappa of 0.63 for IBS and 0.68 for FD. However, this is somewhat below the test-retest coefficient of $82 \%$ found for the English language version of the questionnaire. ${ }^{14}$

In the present study, frequencies of reporting "red flag" symptoms (eg, blood in stools and unintended weight loss) were concordant with previous reports. ${ }^{8,21}$ It is not surprising that the frequency of family history of any GI cancer in our findings was relatively high in each group (25.5 to $28.6 \%$, Table 5 ) since gastric and colon cancers are very common in the Japanese population. ${ }^{22}$ Notably, the red flags are not automatically cause for alarm signs. A separate, benign problem (eg, rectal bleeding caused by hemorrhoids) is often found that explains them. In the present study, no organic abnormality had been detected in the colon for all of patients who were diagnosed as IBS with colonoscopy or barium enema despite the self-reported alarm symptoms on the red flag questionnaire. Whitehead et $\mathrm{al}^{8}$ reported that patients with IBS or other functional bowel disorders are highly likely to report red flag symptoms, suggesting that these symptoms should not be incorporated into the symptom-based Rome criteria as exclusions. Therefore, the "red flag" questionnaires are not meant to be used for the discrimination of patients with FGIDs from patients who have any serious conditions but are used for identifying patients who may require further diagnostic testing to determine whether organic diseases are present.

A limitation of this and all other studies seeking to validate symptom based diagnostic criteria for FGIDs is that there is no biomarker to serve as a gold standard. The approach taken here of comparing questionnaire based diagnoses to medical diagnoses 
made by experienced clinicians has been employed in other studies, but it should be acknowledged that clinicians often disagree about the diagnosis of an FGID in a specific patient. ${ }^{14} \mathrm{~A}$ second study limitation was that estimates of sensitivity and specificity were based on the ability of the Rome III Diagnostic Questionnaire to discriminate healthy controls from patients with FGIDs. This over-estimates the ability to discriminate patients with FGIDs from patients with other gastrointestinal symptoms, which is more important to clinicians than the ability to distinguish patients with FGIDs from healthy controls. Moreover, it is also important to evaluate whether the questionnaire could differentiate different types of FGIDs. Thirdly, we did not investigate whether participants may take any medication for GI and/or the other reasons. It is unlikely that participants selected by physicians may be biased since physicians were asked to exclude FGID patients who had improved their GI symptoms and the mean severity score in IBS patients was considered as a moderate level at the index visit on the IBS-SSS, ${ }^{13}$ which was almost concordant with the previous findings of validation studies for IBS. ${ }^{9,23,24}$ Lastly, IBS patients were significantly younger in age compared with FD patients or control subjects. Younger population might be more educated than older population in Japan. In the further studies, socioeconomic status such as an education level in each subject should be taken into account.

In conclusion, the present findings demonstrate that both the IBS and FD diagnostic modules on the Japanese version of the Rome III diagnostic questionnaire demonstrate good validity and reliability. In addition, our findings support the idea that patient-reported red flag symptoms should not be incorporated into the Rome criteria as exclusions. Thus, this Japanese Rome III Diagnostic Questionnaire can be used for cross-cultural comparisons on functional GI disorders between Japan and other countries.

\section{Acknowledgements}

The authors thank Ms. Caryn Jones, Representative Director, Think SCIENCE K.K. for the back-translation of the instruments. This research was supported by a Grant-in-Aid for Scientific Research from the Ministry of Education, Science, and Culture of Japan, and a Grant-in-Aid for Scientific Research from the Ministry of Health, Welfare, and Labor of Japan. Principal investigator of these grants was Professor Shin Fukudo, Tohoku University.

\section{Supplementary Materials}

Note: To access the supplementary Figure mentioned in this article, visit the online version of Journal of Neurogastroenterology and Motility at http://www.jnmjournal.org/, and at http://dx.doi.org/10.5056/jnm15016.

\section{References}

1. Drossman DA, Corazziari E, Delvaux M, et al. Rome III: The Functional Gastrointestinal Disorders. McLean, VA: Degnon Associates, Inc. 2006.

2. Toner BB, Chang L, Fukudo S, et al. Gender, age, society, culture, and the patient's perspective. In: Drossman DA, Corazziari E, Delvaux M, et al., eds. Rome III: The Functional Gastrointestinal Disorders. McLean, VA: Degnon Associates, Inc. 2006:231-294.

3. Tack J, Talley NJ, Camilleri M, et al. Functional gastroduodenal disorders. In: Drossman DA, Corazziari E, Delvaux M, et al., eds. Rome III: The Functional Gastrointestinal Disorders. McLean, VA: Degnon Associates, Inc. 2006:419-486.

4. Drossman DA, Corazziari E, Talley NJ, Thompson WG, Whitehead WE. Rome II: The Functional Gastrointestinal Disorders. McLean, VA: Degnon Associates, Inc. 2000.

5. Longstreth GF, Thompson WG, Chey WD, Houghton LA, Mearin F, Spiller RC. Functional bowel disorders. In: Drossman DA, Corazziari E, Delvaux M, et al., eds. Rome III: The Functional Gastrointestinal Disorders. McLean, Virginia, USA: Degnon Associates, Inc. 2006:487-555.

6. Heaton KW, Radvan J, Cripps H, Mountford RA, Braddon FE, Hughes AO. Defecation frequency and timing, and stool form in the general population: a prospective study. Gut 1992;33:818-824.

7. Whitehead WE, Validation Working Team in association with the Rome Questionnaire Committee. Development and validation of the Rome III diagnostic questionnaire. In: Drossman DA, Corazziari E, Delvaux M, et al., eds. Rome III: The Functional Gastrointestinal Disorders. McLean, VA: Degnon Associates, Inc; 2006:835-865.

8. Whitehead WE, Palsson OS, Feld AD, et al. Utility of red flag symptom exclusions in the diagnosis of irritable bowel syndrome. Aliment Pharmacol Ther 2006;24:137-146.

9. Shinozaki M, Kanazawa M, Sagami Y, et al. Validation of the Japanese version of the Rome II modular questionnaire and irritable bowel syndrome severity index. J Gastroenterol 2006;41:491-494.

10. Nakajima S, Takahashi K, Sato J, et al. Spectra of functional gastrointestinal disorders diagnosed by Rome III integrative questionnaire in a Japanese outpatient office and the impact of overlapping. J Gastroenterol Hepatol 2010;25(suppl 1): S138-S143.

11. Miwa H. Life style in persons with functional gastrointestinal disorders - large-scale internet survey of lifestyle in Japan. Neurogastroenterol Motil 2012;24:464-471.

12. Sperber AD. Translation and validation of study instruments for cross-cultural research. Gastroenterology 2004;126(suppl 1):S124S128.

13. Francis CY, Morris J, Whorwell PJ. The irritable bowel severity 
scoring system: a simple method of monitoring irritable bowel syndrome and its progress. Aliment Pharmacol Ther 1997;11:395-402.

14. Whitehead WE, Drossman DA. Validation of symptom-based diagnostic criteria for irritable bowel syndrome: a critical review. Am J Gastroenterol 2010;105:814-820.

15. Lee YY, Waid A, Tan HJ, Chua SB, Whitehead WE. Validity and reliability of the Malay-language translation of the Rome III Diagnostic Questionnaire for irritable bowel syndrome. J Gastroenterol Hepatol 2012;27:746-750.

16. Reisswitz PS, Mazzoleni LE, Sander GB, Francisconi CF. Portuguese validation of the Rome III diagnostic questionnaire for functional dyspepsia. Arq Gastroenterol 2010;47:354-360.

17. Goshal UC, Gwee K, Chen M, et al. Development, translation and validation of enhanced Asian Rome III Questionnaire for diagnosis of functional bowel diseases in major Asian languages: a Rome Foundation-Asian Neurogastroenterology and Motility Association working team report. J Neurogastroenterol Motil 2015;21:83-92.

18. Whitehead WE, Palsson OS, Levy RL, et al. Agreement of Rome criteria with clinical diagnosis of irritable bowel (IBS). Gastroenterology
2003;124:A397.

19. Vandvik PO, Aabakken L, Farup PG. Diagnosing irritable bowel syndrome: poor agreement between general practitioners and the Rome II criteria. Scand J Gastroenterol 2004;39:448-453.

20. Song KH, Jung HK, Min BH, et al. Development and validation of the Korean Rome III Questionnaire for diagnosis of functional gastrointestinal disorders. J Neurogastroenterol Motil 2013;19:509-515.

21. Hammer J, Eslick GD, Howell SC, Altiparmak E, Talley NJ. Diagnostic yield of alarm features in irritable bowel syndrome and functional dyspepsia. Gut 2004;53:666-672.

22. Nishino Y, Suzuki Y, Ohmori K, et al. Cancer incidence profiles in the Miyagi Cohort Study. J Epidemiol 2004;14(suppl 1):S7-S1 1.

23. Spiegel B, Bolus R, Harris LA, et al. Measuring irritable bowel syndrome patient-reported outcomes with an abdominal pain numeric rating scale. Aliment Pharmacol Ther 2009;30:1159-1170.

24. Saigo T, Tayama J, Hamaguchi T, et al. Gastrointestinal specific anxiety in irritable bowel syndrome: validation of the Japanese version of the visceral sensitivity index for university students. Biopsychosoc Med 2014;8:10. 